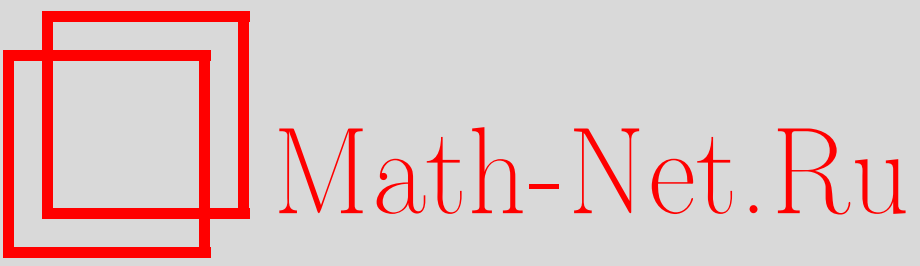

Л. Фраппат, П. Сорба, А. Сциаррино, Квантовые группы и генетический код, ТМФ, 2001, том 128, номер 1, 27-42

DOI: https://doi.org/10.4213/tmf480

Использование Общероссийского математического портала Math-Net.Ru подразумевает, что вы прочитали и согласны с пользовательским соглашением

http://www.mathnet.ru/rus/agreement

Параметры загрузки:

IP : 3.93 .64 .190

26 апреля 2023 г., 15:19:33 
ТЕОРЕТИЧЕСКАЯ

И МАТЕМАТИЧЕСКАЯ

ФИЗИКА

Том 128, № 1

июль, 2001

(c) 2001 г. $\quad$ Л. Фраппат* ${ }^{*}$ П. Сорба* А. Сциаррино $^{\dagger}$

\title{
КВАНТОВЫЕ ГРУППЫ И ГЕНЕТИЧЕСКИЙ КОД
}

\begin{abstract}
Квантовая алгебра $\mathcal{U}_{q}(s l(2) \oplus s l(2))$ рассматривается в пределе $q \rightarrow 0$ как алгебра симметрий генетического кода. Триплеты нуклеотидов (кодоны) в цепях ДНК классифицированы с помощью кристальных базисов. Эту конструкцию можно сравнить с кварковой классификацией барионов в физике частиц. Главное отличие от последней состоит в том, что кристальный базис задает естественное упорядочение элементарных составляющих состояния модели. Оператор, задающий соответствие между кодонами и аминокислотами, строится с помощью алгебры симметрий: парам кодонов, отвечающим одной и той же аминокислоте, ставится в соответствие одно и то же собственное число, а парам, отвечающим разным аминокислотам, - разные собственные числа. Выводится и сравнивается с экспериментальными данными ряд соотношений для физико-химических свойств аминокислот. Квартетные и секстетные комбинации кодонов естественным образом появляются в описываемой модели.
\end{abstract}

\section{1. ВВЕДЕНИЕ}

Точное соответствие между триплетами нуклеотидов в цепочках дезоксирибонуклеиновой кислоты (ДНК) и аминокислотами называется генетическим кодом [1]. Макромолекула ДНК, управляюшая синтезом белков (основных органических соединений живых организмов), состоит из двух линейных цепочек нуклеотидов, закрученных в знаменитую двойную спираль. Каждый нуклеотид содержит одну из четырех элементарных структур (оснований): аденин $(\mathrm{A})$ или гуанин $(\mathrm{G})$, получаемые из пурина, или цитозин $(\mathrm{C})$ или тимин (T), получаемые из пиримидина. Молекула ДНК содержится в ядре клетки, а сообшаюшая (или матричная) рибонуклеиновая кислота (мРНК) переносит генетическую информацию в цитоплазму. Операция перехода на мРНК информации, храняшейся в ДНК, называется транскрипцией, при этом основания ДНК А, G, С и Т порождают соответствуюшие основания $\mathrm{U}, \mathrm{C}, \mathrm{G}$ и $\mathrm{A}$ (через $\mathrm{U}$ обозначена элементарная структура урацила) молекулы мРНК. Кодон - это упорядоченная последовательность из трех оснований; всего имеется $4 \times 4 \times 4$ различных кодонов. В генетическом коде каж-

*Laboratoire d'Annecy-le-Vieux de Physique Théorique LAPTH CNRS, UMR 5108, associée à l'Université de Savoie BP 110, F-74941 Annecy-le-Vieux CEDEX, France

${ }^{\dagger}$ Dipartimento di Scienze Fisiche, Università di Napoli Federico II; I.N.F.N., Sezione di Napoli, Italy, Complesso di Monte, S. Angelo, Via Cintia, I-80126 Napoli, Italy 
ТАБЛИЦА 1

Евкариотический код: верхний индекс обозначает различные неприводимые представления

\begin{tabular}{|c|c|c|c|c|c|c|c|}
\hline Кодон & А.к. & $J_{\mathrm{H}}$ & $J_{\mathrm{V}}$ & Кодон & A.к. & $J_{\mathrm{H}}$ & $J_{\mathrm{V}}$ \\
\hline $\mathrm{CCC}$ & Pro & $3 / 2$ & $3 / 2$ & UCC & Ser & $3 / 2$ & $3 / 2$ \\
\hline $\mathrm{CCU}$ & Pro & $(1 / 2$ & $3 / 2)^{1}$ & $\mathrm{UCU}$ & Ser & $(1 / 2$ & $3 / 2)^{1}$ \\
\hline CCG & Pro & $(3 / 2$ & $1 / 2)^{1}$ & UCG & Ser & $(3 / 2$ & $1 / 2)^{1}$ \\
\hline CCA & Pro & $(1 / 2$ & $1 / 2)^{1}$ & $\mathrm{UCA}$ & Ser & $(1 / 2$ & $1 / 2)^{1}$ \\
\hline CUC & Leu & $(1 / 2$ & $3 / 2)^{2}$ & UUC & Phe & $3 / 2$ & $3 / 2$ \\
\hline CUU & Leu & $(1 / 2$ & $3 / 2)^{2}$ & UUU & Phe & $3 / 2$ & $3 / 2$ \\
\hline CUG & Leu & $(1 / 2$ & $1 / 2)^{3}$ & UUG & Leu & $(3 / 2$ & $1 / 2)^{1}$ \\
\hline CUA & Leu & $(1 / 2$ & $1 / 2)^{3}$ & UUA & Leu & $(3 / 2$ & $1 / 2)^{1}$ \\
\hline CGC & Arg & $(3 / 2$ & $1 / 2)^{2}$ & UGC & Cys & $(3 / 2$ & $1 / 2)^{2}$ \\
\hline $\mathrm{CGU}$ & Arg & $(1 / 2$ & $1 / 2)^{2}$ & $\mathrm{UGU}$ & Cys & $(1 / 2$ & $1 / 2)^{2}$ \\
\hline CGG & Arg & $(3 / 2$ & $1 / 2)^{2}$ & UGG & Trp & $(3 / 2$ & $1 / 2)^{2}$ \\
\hline CGA & Arg & $(1 / 2$ & $1 / 2)^{2}$ & UGA & Ter & $(1 / 2$ & $1 / 2)^{2}$ \\
\hline $\mathrm{CAC}$ & His & $(1 / 2$ & $1 / 2)^{4}$ & UAC & Tyr & $(3 / 2$ & $\overline{1 / 2)^{2}}$ \\
\hline CAU & His & $(1 / 2$ & $1 / 2)^{4}$ & $\mathrm{UAU}$ & Tyr & $(3 / 2$ & $1 / 2)^{2}$ \\
\hline CAG & Gln & $(1 / 2$ & $1 / 2)^{4}$ & UAG & Ter & $(3 / 2$ & $1 / 2)^{2}$ \\
\hline CAA & Gln & $(1 / 2$ & $1 / 2)^{4}$ & UAA & Ter & $(3 / 2$ & $1 / 2)^{2}$ \\
\hline GCC & Ala & $3 / 2$ & $3 / 2$ & $\mathrm{ACC}$ & Thr & $3 / 2$ & $3 / 2$ \\
\hline GCU & Ala & $(1 / 2$ & $3 / 2)^{1}$ & $\mathrm{ACU}$ & Thr & $(1 / 2$ & $3 / 2)^{1}$ \\
\hline GCG & Ala & $(3 / 2$ & $1 / 2)^{1}$ & $\mathrm{ACG}$ & Thr & $(3 / 2$ & $1 / 2)^{1}$ \\
\hline GCA & Ala & $(1 / 2$ & $1 / 2)^{1}$ & $\mathrm{ACA}$ & Thr & $(1 / 2$ & $1 / 2)^{1}$ \\
\hline GUC & $\mathrm{Val}$ & $(1 / 2$ & $3 / 2)^{2}$ & $\mathrm{AUC}$ & Ile & $3 / 2$ & $3 / 2$ \\
\hline GUU & Val & $(1 / 2$ & $3 / 2)^{2}$ & AUU & Ile & $3 / 2$ & $3 / 2$ \\
\hline GUG & Val & $(1 / 2$ & $1 / 2)^{3}$ & AUG & Met & $(3 / 2$ & $1 / 2)^{1}$ \\
\hline GUA & Val & $(1 / 2$ & $1 / 2)^{3}$ & AUA & Ile & $(3 / 2$ & $1 / 2)^{1}$ \\
\hline GGC & Gly & $3 / 2$ & $3 / 2$ & $\mathrm{AGC}$ & Ser & $3 / 2$ & $3 / 2$ \\
\hline GGU & Gly & $(1 / 2$ & $3 / 2)^{1}$ & $\mathrm{AGU}$ & Ser & $(1 / 2$ & $3 / 2)^{1}$ \\
\hline GGG & Gly & $3 / 2$ & $3 / 2$ & $\mathrm{AGG}$ & Arg & $3 / 2$ & $3 / 2$ \\
\hline GGA & Gly & $(1 / 2$ & $3 / 2)^{1}$ & AGA & Arg & $(1 / 2$ & $3 / 2)^{1}$ \\
\hline GAC & Asp & $(1 / 2$ & $3 / 2)^{2}$ & $\mathrm{AAC}$ & Asn & $3 / 2$ & $3 / 2$ \\
\hline GAU & Asp & $(1 / 2$ & $3 / 2)^{2}$ & $\mathrm{AAU}$ & Asn & $3 / 2$ & $3 / 2$ \\
\hline GAG & Glu & $(1 / 2$ & $3 / 2)^{2}$ & $\mathrm{AAG}$ & Lys & $3 / 2$ & $3 / 2$ \\
\hline GAA & Glu & $(1 / 2$ & $3 / 2)^{2}$ & $\mathrm{AAA}$ & Lys & $3 / 2$ & $3 / 2$ \\
\hline
\end{tabular}


дый из 64 кодонов либо связан через рибосому со своей аминокислотой, либо используется как прерываюший сигнал. Кодоны, выполняюшие последнюю функцию, называются бессмысленными или останавливающими кодонами; их роль и состоит в прерывании биосинтеза. В белках живых систем встречаются двадшать различных аминокислот, а потому одной и той же аминокислоте могут отвечать различные кодоны, т.е. генетический код вырож ден. В стандартном евкариотическом подходе (табл. 1) кодоны собраны в мультиплеты, каждый из которых соответствует одной аминокислоте.

\section{2. МОДЕЛЬ [2]}

Четыре нуклеотида будут интерпретироваться как базисные состояния $(1 / 2,1 / 2)$ представления квантовой алгебры $\mathcal{U}_{q}(s l(2) \oplus s l(2))$ в пределе $q \rightarrow 0$. Кодон - это элемент тензорного произведения трех таких представлений размерности четыре. Этот подход напоминает теоретико-групповую классификацию барионов, принятую в физике частиц. Барионы состоят из трех кварков, а в данном случае имеются четыре строительных блока: нуклеотиды A, C, G и T (U). Существенное отличие состоит также и в том, что кодон представляет собой упорядоченный набор трех нуклеотидов, в то время как кварки в барионах неупорядочены: из нуклеотидов A, А и U можно составить три различных кодона (именно AAU, AUA и UAA), в то время как протон представляет собой взвешенную комбинацию двух $u$ и одного $d$ кварка (именно $|p\rangle \sim|u u d\rangle+|u d u\rangle+|d u u\rangle$ ), в которой также предполагается наличие спиновой структуры.

Построение таких чистых состояний возможно в любой алгебре $\mathcal{U}_{q \rightarrow 0}(\mathcal{G})$, где $\mathcal{G}-$ это любая (полу)простая классическая алгебра Ли, поскольку любая такая алгебра допускает сушествование специального базиса, называемого кристальным базисом, в любом конечномерном представлении $\mathcal{G}$. Однако алгебра $\mathcal{G}=s u(2) \oplus s u(2)$ представляется наиболее естественной для описания явления в данной работе. Прежде всего, кажется естественным отождествить четыре нуклеотида с элементами фундаментального представления $\mathcal{G}$. Кроме того, правило комплементарности операции считывания ДНКмРНК предполагает, что элементам пар (A,T/U) и $(\mathrm{C}, \mathrm{G})$ должны быть присвоены противоположные квантовые числа. Аналогично можно попытаться алгебраически представить различие между пуриновыми $(\mathrm{A}, \mathrm{G})$ и пиримидиновыми $(\mathrm{C}, \mathrm{T} / \mathrm{U})$ основаниями. Таким образом, выбирая представление $(1 / 2,1 / 2)$ групшы $S U(2) \times S U(2)$ и маркируя с помошью \pm базисные векторы, отвечающие собственным значениям $\pm 1 / 2$ генераторов $J_{3}$ двух алгебр $s l(2)$, предположим, что “биологическая” спиновая структура имеет вид

$$
\begin{aligned}
& \mathrm{C} \equiv(+,+) \stackrel{s u(2)_{\mathrm{H}}}{\longrightarrow} \mathrm{U} \equiv(-,+) \\
& s u(2)_{\mathrm{V}} \uparrow \\
& \mathrm{G} \equiv(+,-) \underset{s u(2)_{\mathrm{H}}}{\longrightarrow} \mathrm{A} \equiv(-,-),
\end{aligned}
$$

где нижние индексы H (horizontal) и V (vertical) явно указывают действия групп.

Рассмотрим теперь представления $\mathcal{U}_{q \rightarrow 0}(\mathcal{G})$ или, более точно, их кристальные базисы. Предел $q \rightarrow 0$ деформированной (квантовой) алгебры в статистической механике может интерпретироваться как предел абсолютного нуля температуры в решеточной 
модели. В пределе $\mathcal{U}_{q \rightarrow 0}(\mathcal{G})$ простые корневые векторы $e_{i}$ и $f_{i}$ алгебры $\mathcal{U}_{q}(\mathcal{G})$ переходят в операторы $\tilde{e}_{i}$ и $\tilde{f}_{i}, i=1, \ldots, \operatorname{rank} \mathcal{G}$, и в $\mathcal{U}_{q}(\mathcal{G})$-модуле вводится специальный базис, называемый кристальным базисом. Операторы $\tilde{e}_{i}$ и $\tilde{f}_{i}$ действуют в этом базисе особенно простым образом: для любой пары векторов $u$ и $v$ из кристального базиса $\mathcal{B}$ сооношение $u=\tilde{e}_{i} v$ выполняется тогда и только тогда, когда $v=\tilde{f}_{i} u$. Тензорное произведение двух представлений в кристальном базисе дается следующей конструкцией [3]. Пусть $\mathcal{B}_{1}$ и $\mathcal{B}_{2}$ суть кристальные базисы $\mathcal{U}_{q \rightarrow 0}(\mathcal{G})$-модулей $M_{1}$ и $M_{2}$ и $u \in \mathcal{B}_{1}, v \in \mathcal{B}_{2}$. Тогда

$$
\begin{aligned}
& \tilde{f}_{i}(u \otimes v)= \begin{cases}\tilde{f}_{i} u \otimes v, & \text { если } \exists n \geqslant 1 \text { такое, что } \tilde{f}_{i}^{n} u \neq 0 \text { и } \tilde{e}_{i}^{n} v=0, \\
u \otimes \tilde{f}_{i} v \quad \text { в противном случае, }\end{cases} \\
& \tilde{e}_{i}(u \otimes v)= \begin{cases}u \otimes \tilde{e}_{i} v, & \text { если } \exists n \geqslant 1 \text { такое, что } \tilde{e}_{i}^{n} v \neq 0 \text { и } \tilde{f}_{i}^{n} u=0, \\
\tilde{e}_{i} u \otimes v & \text { в противном случае. }\end{cases}
\end{aligned}
$$

Выберем кристальный базис, который сушествует лишь в пределе $q \rightarrow 0$. Известно, что порядок вхождения нуклеотидов в кодон исключительно важен (например, $\mathrm{CCU} \rightarrow \operatorname{Pro}, \mathrm{CUC} \rightarrow$ Leu и UCC $\rightarrow \mathrm{Ser})$. Будем рассматривать кодоны как составные состояния (элементарных) нуклеотидов, но это-то как раз и невозможно сделать в подходе (супер)алгебр Ли. В самом деле, составные состояния, получаемые как тензорные произведения фундаментальных неприводимых представлений в теории алгебр Ли, суть линейные комбинации элементарных состояний; свойства симметрий этих комбинаций определяются структурой тензорных произведений (т.е. структурой соответствуюшей таблицы Юнга для $s l(n))$. Напротив, математическая структура кристального базиса позволяет строить иистые составные состояния, задаваемые простым порядком вхождения множителей. Но для того чтобы ввести такой базис, надо взять предел $q \rightarrow 0$. Заметим, что в этом пределе не остается ни алгебры Ли, ни ее универсальной деформированной обертываюшей алгебры.

Для построения такого базиса интерпретируем четыре состояния $\mathrm{C}, \mathrm{U}, \mathrm{G}$ и $\mathrm{A}$, определенные диаграммой (1), как составляющие кристального базиса $(1 / 2,1 / 2)$-модуля при $\mathcal{U}_{q \rightarrow 0}(s l(2) \oplus s l(2))$. Кодон задается тензорным произведением трех $(1 / 2,1 / 2)$ представлений алгебры $\mathcal{U}_{q \rightarrow 0}(s l(2) \oplus s l(2))$. Однако хорошо известно (см. табл. 1$)$, что в мультиплете кодонов, отвечающих некоторой аминокислоте, первые два нуклеотида, как правило, одни и те же (свойство стабильности), а вырождение идет в основном по третьему нуклеотиду, поэтому удобно представлять кодон как $(2+1)$-состояние, а не просто как триплет. Рассмотрим теперь более детально первое тензорное произведение:

$$
\left(\frac{1}{2}, \frac{1}{2}\right) \otimes\left(\frac{1}{2}, \frac{1}{2}\right)=(1,1) \oplus(1,0) \oplus(0,1) \oplus(0,0),
$$

в котором индексы $j=0,1 / 2,1$ внутри скобок обозначают $(2 j+1)$-мерные представления группы $S U(2)$. Из правил тензорного произведения следует диаграмма

$$
\begin{aligned}
& \rightarrow s u(2)_{\mathrm{H}} \quad(0,0) \quad(\mathrm{CA}) \\
& \downarrow \\
& s u(2)_{\mathrm{V}} \\
& (0,1)\left(\begin{array}{l}
\mathrm{CU} \\
\mathrm{GU} \\
\mathrm{GA}
\end{array}\right) \\
& (1,0) \quad(\mathrm{CG} \quad \mathrm{UG} \quad \mathrm{UA}) \\
& (1,1)\left(\begin{array}{ccc}
\mathrm{CC} & \mathrm{UC} & \mathrm{UU} \\
\mathrm{GC} & \mathrm{AC} & \mathrm{AU} \\
\mathrm{GG} & \mathrm{AG} & \mathrm{AA}
\end{array}\right) \text {. }
\end{aligned}
$$


Из табл. 1 следует, что динуклеотидным состояниям, образованным двумя первыми нуклеотидами кодона, могут быть поставлены в соответствие квадруплеты, дублеты или синглеты кодонов, отвечающих одной и той же аминокислоте. Возникающие при этом также секстеты и триплеты рассматриваются как соответствующие комбинации квадруплет-дублет и дублет-синглет.

Определим “заряд” $Q$ динуклеотидного состояния:

$$
Q=J_{3, \mathrm{H}}+\frac{1}{4} C_{\mathrm{V}}\left(J_{3, \mathrm{~V}}+1\right)-\frac{1}{4},
$$

где $J_{3, \alpha}(\alpha=\mathrm{H}, \mathrm{V})$ - это диагонализованный генератор $s l(2)_{\alpha}$, а $C_{\mathrm{V}}$ - оператор Казимира алгебры $\mathcal{U}_{q \rightarrow 0}\left(s l(2)_{\mathrm{V}}\right)$; собственное значение этого оператора в $j$-представлении равно $j(j+1)$. Динуклеотидные состояния разбиваются на два октета по отношению к заряду $Q$ : восемь сильных динуклеотидов ассоциируются с квадруплетами (в том числе входящими в секстеты) кодонов, удовлетворяющих условию $Q>0$, а восемь слабыx динуклеотидов ассоциируются с дублетами (в том числе входящими в триплеты) и, наконец, с синглетами кодонов, удовлетворяюших условию $Q<0$. Заметим, что замена $\mathrm{C} \leftrightarrow \mathrm{A}$ и $\mathrm{U} \leftrightarrow \mathrm{G}$, которая эквивалентна изменению знака $J_{3, \alpha}$ или отражению относительно диагонали в (1), переводит восемь сильных динуклеотидов в слабые и наоборот.

Троекратное тензорное произведение представляется в терминах неприводимых представлений $\mathcal{U}_{q \rightarrow 0}(s l(2) \oplus \operatorname{sl}(2))$ в виде

$$
\left(\frac{1}{2}, \frac{1}{2}\right) \otimes\left(\frac{1}{2}, \frac{1}{2}\right) \otimes\left(\frac{1}{2}, \frac{1}{2}\right)=\left(\frac{3}{2}, \frac{3}{2}\right) \oplus 2\left(\frac{3}{2}, \frac{1}{2}\right) \oplus 2\left(\frac{1}{2}, \frac{3}{2}\right) \oplus 4\left(\frac{1}{2}, \frac{1}{2}\right)
$$

и для соответствующих аминокислот приведено во второй и четвертой колонках табл. 1.

\section{3. СЧИТЫВАЮШИЙ, ИЛИ РИБОСОМНЫЙ, ОПЕРАТОР $\mathcal{R}$ [2], [4]}

Из табл. 1 ясно, что кодоны, отвечающие одной и той же аминокислоте, не объединяются в один неприводимый мультиплет. Однако можно построить оператор $\mathcal{R}$, принадлежаший алгебре $\mathcal{U}_{q \rightarrow 0}(s l(2) \oplus \operatorname{sl}(2))$, который при действии на кодоны определяет различные генетические коды следующим образом:

- Собственные числа двух кодонов под действием оператора $\mathcal{R}$ совпадают тогда и только тогда, когда эти кодоны порождают одну и ту же аминокислоту. Такой оператор $\mathcal{R}$ называется считывающим оператором.

Как известно, сушествуют различные генетические коды: авторам известно двенадцать кодов, относяшихся к группе евкариотических. Предполагается, что различия меж ду генетическими кодами относительно малы, и можно определить “прототип” оператора $\mathcal{R}$ так, чтобы "настояший” оператор $\mathcal{R}$ для каждого отдельного кода представлялся суммой прототипного оператора и "возмушения", которое и отличает один оператор $\mathcal{R}$ от другого. Прототипный код устанавливает квартетную/дублетную структуру различных кодонов. В этом подходе различные считывающие операторы (каждый из которых отвечает своему коду) обладают общим свойством: каждой аминокислоте они присваивают одно и то же собственное число, независимо от используемого кода. 
ТАБЛИЦА 2

\section{Собственные значения считывающих}

операторов аминокислот

\begin{tabular}{c|c||c|c||c|c} 
A.к. & Значение $\mathcal{R}$ & А.к. & Значение $\mathcal{R}$ & А.к. & Значение $\mathcal{R}$ \\
\hline Ala & $-c+3$ & Gly & $-c+5$ & Pro & $-c-1$ \\
Arg & $-c+1$ & His & $-3 c+1$ & Ser & $3 c-1$ \\
Asn & $9 c+5$ & Ile & $5 c+9$ & Thr & $3 c+3$ \\
Asp & $5 c+5$ & Leu & $c-1$ & Trp & $3 c-5$ \\
Cys & $3 c+7$ & Lys & $17 c+5$ & Tyr & $c+1$ \\
Gln & $5 c+1$ & Met & $5 c-3$ & Val & $c+3$ \\
Glu & $13 c+5$ & Phe & $-7 c-1$ & Ter & $9 c+1$
\end{tabular}

Прототипный считывающий оператор $\mathcal{R}$ имеет вид

$\mathcal{R}_{\text {prot }}=\frac{4}{3} c_{1} C_{\mathrm{H}}+\frac{4}{3} c_{2} C_{\mathrm{V}}-4 c_{1} \mathcal{P}_{\mathrm{H}} J_{\mathrm{H}, 3}-4 c_{2} \mathcal{P}_{\mathrm{V}} J_{\mathrm{V}, 3}+\left(-8 c_{1} \mathcal{P}_{\mathrm{D}}+\left(8 c_{1}+12 c_{2}\right) \mathcal{P}_{\mathrm{S}}\right) J_{\mathrm{V}, 3}$.

Определим использованные здесь величины. Операторы $J_{\alpha, 3}(\alpha=\mathrm{H}, \mathrm{V})$ суть третьи компоненты операторов полного спина из алгебры $\mathcal{U}_{q \rightarrow 0}(s l(2) \oplus s l(2))$. Оператор $C_{\alpha}-$ это оператор Казимира алгебры $\mathcal{U}_{q \rightarrow 0}\left(s l(2)_{\alpha}\right)$ в кристальном базисе. Этот оператор коммутирует с $J_{\alpha \pm}$ и с $J_{\alpha, 3}$, и его собственное значение в векторном базисе неприводимого представления старшего веса $J$ равно $J(J+1)$, т.е. оно совпадает с собственным значением недеформированного стандартного оператора Казимира второго порядка для алгебры $s l(2)$. Явное выражение для последнего оператора имеет вид

$$
C_{\alpha}=\left(J_{\alpha, 3}\right)^{2}+\frac{1}{2} \sum_{n \in \mathbb{Z}_{+}} \sum_{k=0}^{n}\left(J_{\alpha-}\right)^{n-k}\left(J_{\alpha+}\right)^{n}\left(J_{\alpha-}\right)^{k}
$$

Заметим, что операторы Казимира при $s l(2)_{q \rightarrow 0}$ задаются бесконечными степенными рядами по $J_{\alpha \pm}$. Тем не менее только лишь конечное число членов этих рядов дают неисчезающий вклад в случае каждого конечного неприводимого представления. Операторы $\mathcal{P}_{\mathrm{H}}$ и $\mathcal{P}_{\mathrm{V}}$ суть проекционные операторы, задаваемые формулами:

$$
\mathcal{P}_{\mathrm{H}}=J_{\mathrm{H}+}^{d} J_{\mathrm{H}-}^{d}, \quad \mathcal{P}_{\mathrm{V}}=J_{\mathrm{V}+}^{d} J_{\mathrm{V}-}^{d}
$$


где операторы $J_{\alpha, \pm}^{d}$ относятся к динуклеотидам, т.е. к первым двум нуклеотидам кодона. Проекционные операторы $\mathcal{P}_{\mathrm{D}}$ и $\mathcal{P}_{\mathrm{S}}$ тогда задаются формулами

$$
\begin{aligned}
\mathcal{P}_{\mathrm{D}}= & \left(1-J_{\mathrm{V}+}^{d} J_{\mathrm{V}-}^{d}\right)\left(J_{\mathrm{H}+}^{d} J_{\mathrm{H}-}^{d}\right)\left(J_{\mathrm{H}-}^{d} J_{\mathrm{H}+}^{d}\right)+\left(1-J_{\mathrm{H}+}^{d} J_{\mathrm{H}-}^{d}\right)\left(1-J_{\mathrm{V}+}^{d} J_{\mathrm{V}-}^{d}\right)+ \\
& +\left(1-J_{\mathrm{H}+}^{d} J_{\mathrm{H}-}^{d}\right)\left(J_{\mathrm{V}+}^{d} J_{\mathrm{V}-}^{d}\right)\left(J_{\mathrm{H}-}^{d} J_{\mathrm{H}+}^{d}\right), \\
\mathcal{P}_{\mathrm{S}}= & \left(J_{\mathrm{H}-}^{d} J_{\mathrm{H}+}^{d}\right)\left[\left(J_{\mathrm{H}+}^{d} J_{\mathrm{H}-}^{d}\right)\left(1-J_{\mathrm{V}+}^{d} J_{\mathrm{V}-}^{d}\right)+\left(J_{\mathrm{V}+}^{d} J_{\mathrm{V}-}^{d}\right)\left(J_{\mathrm{V}-}^{d} J_{\mathrm{V}+}^{d}\right)\left(1-J_{\mathrm{H}+}^{d} J_{\mathrm{H}-}^{d}\right)\right] .
\end{aligned}
$$

Явное выражение для оператора евкариотического кода имеет вид

$$
\begin{aligned}
\mathcal{R}_{\mathrm{EC}}= & \frac{4}{3} c_{1} C_{\mathrm{H}}+\frac{4}{3} c_{2} C_{\mathrm{V}}-4 c_{1} \mathcal{P}_{\mathrm{H}} J_{\mathrm{H}, 3}-4 c_{2} \mathcal{P}_{\mathrm{V}} J_{\mathrm{V}, 3}+ \\
& +\left(-8 c_{1} \mathcal{P}_{\mathrm{D}}+\left(8 c_{1}+12 c_{2}\right) \mathcal{P}_{\mathrm{S}}\right) J_{\mathrm{V}, 3}+\left(-4 c_{1}+14 c_{2}\right) \mathcal{P}_{\mathrm{AG}}\left(\frac{1}{2}-J_{\mathrm{V}, 3}^{(3)}\right)+ \\
& +\left[12 c_{2} \mathcal{P}_{\mathrm{AU}}+\left(6 c_{1}+6 c_{2}\right) \mathcal{P}_{\mathrm{UG}}\right]\left(\frac{1}{2}-J_{\mathrm{V}, 3}^{(3)}\right)\left(\frac{1}{2}-J_{\mathrm{H}, 3}^{(3)}\right),
\end{aligned}
$$

где проекционные операторы $\mathcal{P}_{\mathrm{AG}}, \mathcal{P}_{\mathrm{AU}}$ и $\mathcal{P}_{\mathrm{UG}}$ задаются следующими формулами:

$$
\begin{aligned}
& \mathcal{P}_{\mathrm{AG}}=\left(J_{\mathrm{H}+}^{d} J_{\mathrm{H}-}^{d}\right)\left(J_{\mathrm{H}-}^{d} J_{\mathrm{H}+}^{d}\right)\left(1-J_{\mathrm{V}+}^{d} J_{\mathrm{V}-}^{d}\right)\left(J_{\mathrm{V}-}^{d} J_{\mathrm{V}+}^{d}\right), \\
& \mathcal{P}_{\mathrm{AU}}=\left(1-J_{\mathrm{H}+}^{d} J_{\mathrm{H}-}^{d}\right)\left(J_{\mathrm{H}-}^{d} J_{\mathrm{H}+}^{d}\right)\left(J_{\mathrm{V}+}^{d} J_{\mathrm{V}-}^{d}\right)\left(J_{\mathrm{V}-}^{d} J_{\mathrm{V}+}^{d}\right), \\
& \mathcal{P}_{\mathrm{UG}}=\left(J_{\mathrm{H}+}^{d} J_{\mathrm{H}-}^{d}\right)\left(J_{\mathrm{H}-}^{d} J_{\mathrm{H}+}^{d}\right)\left(1-J_{\mathrm{V}+}^{d} J_{\mathrm{V}-}^{d}\right)\left(1-J_{\mathrm{V}-}^{d} J_{\mathrm{V}+}^{d}\right) .
\end{aligned}
$$

Наконец, собственные значения считывающих операторов аминокислот (после масштабного преобразования и введения безразмерной константы $\left.c \equiv c_{1} / c_{2}\right)$ приведены в табл. 2.

Заметим, что $\mathcal{R}(c)$ можно использовать при всех вешественных $c$, кроме тех, которые задают одно и то же собственное число для кодонов, соответствующих различным аминокислотам. Эти запрешенные значения следуюшие:

$$
\begin{aligned}
& -7, \quad-5, \quad-4, \quad-3, \quad-\frac{5}{2}, \quad-\frac{7}{3}, \quad-2, \quad-\frac{5}{3}, \\
& -\frac{3}{2}, \quad-\frac{4}{3}, \quad-1, \quad-\frac{5}{6}, \quad-\frac{4}{5}, \quad-\frac{3}{4}, \quad-\frac{5}{7}, \quad-\frac{2}{3}, \\
& -\frac{3}{5}, \quad-\frac{1}{2}, \quad-\frac{3}{7}, \quad-\frac{2}{5}, \quad-\frac{3}{8}, \quad-\frac{1}{3}, \quad-\frac{3}{10}, \quad-\frac{2}{7} \text {, } \\
& -\frac{1}{4}, \quad-\frac{2}{9}, \quad-\frac{1}{5}, \quad-\frac{1}{6}, \quad-\frac{1}{7}, \quad-\frac{1}{8}, \quad-\frac{1}{9}, \quad 0 \text {, } \\
& \frac{1}{7}, \quad \frac{1}{6}, \quad \frac{1}{5}, \quad \frac{1}{4}, \quad \frac{1}{3}, \quad \frac{2}{5}, \quad \frac{1}{2}, \quad \frac{2}{3}, \\
& 1, \quad \frac{4}{3}, \quad \frac{3}{2}, \quad 2, \quad \frac{5}{2}, \quad 3, \quad 4, \quad 5 .
\end{aligned}
$$

Выражения для считывающих операторов $\mathcal{R}$, отвечающих другим кодам, и их детальное описание содержатся в работе [4]. Отметим еще раз, что все различные считывающие операторы определяют одно и то же собственное число для каждой аминокислоты.

2 Теоретическая и математическая физика, т. 128, № 1, 2001 г. 


\section{4. ФИЗИКО-ХИМИЧЕСКИЕ СВОЙСТВА ДИНУКЛЕОТИДОВ И АМИНОКИСЛОТ}

Исследуемая модель, в которой нуклеотиды характеризуются квантовыми числами, оказывается хорошо приспособленной для получения формул, выражающих биофизические свойства. Начнем с исследования двух величин, зависящих от пары нуклеотидов. Эти величины - это относительные гидрофильность $R_{f}$ и гидрофобность $R_{x}$ динуклеотидов. После этого вычислим выделение энергии при спаривании оснований в двойной спирали РНК [4].

4.1. Гидрофильность. Подгоним теперь значения относительных гидрофильностей $R_{f}$ шестнадцати динуклеозидных монофосфатов [5] с помощью четырехпараметрического оператора

$$
R_{f}=\alpha_{0}+\alpha_{1} C_{\mathrm{V}}+\alpha_{2} J_{3 \mathrm{~V}}^{d}+\alpha_{3} \sum_{i=1,2}\left(J_{3 \mathrm{H}}^{i}+J_{3 \mathrm{~V}}^{i}\right)\left(J_{3 \mathrm{H}}^{i}+J_{3 \mathrm{~V}}^{i}-1\right)
$$

(множитель при $\alpha_{3}$ равен четырем для $\mathrm{AA}$, двум для $\mathrm{CA}, \mathrm{GA}$ и UA и нулю для остальных динуклеотидов). Используя метод наименьших квадратов, можно получить наилучшее приближение для коэффициентов:

$$
\alpha_{0}=0.135, \quad \alpha_{1}=0.036, \quad \alpha_{2}=0.147, \quad \alpha_{3}=-0.016 \text {. }
$$

Среднее отклонение четырехпараметрической оценки (2) от экспериментальных значений равно 0.027. Экспериментальные и подгоночные значения коэффициентов гидрофильности $R_{f}$ динуклеозидов собраны в табл. $3^{1)}$.

ТАБЛИЦА 3

\section{Относительные гидрофильности $R_{f}$ динуклеозидов}

$$
\left.\begin{array}{c}
{\left[\begin{array}{r}
\mathrm{CA}_{0.103}^{0.083}
\end{array}\right] \quad\left[\begin{array}{rrr}
\mathrm{CG}_{0.135}^{0.146} & \mathrm{UG}_{0.135}^{0.359} & \mathrm{UA}_{0.103}^{0.090}
\end{array}\right]} \\
\mathrm{GU}_{0.354}^{0.224} \\
0.207 \\
\mathrm{GA}_{0.028}^{0.035}
\end{array}\right] \quad\left[\begin{array}{rrr}
\mathrm{CC}_{0.354}^{0.349} & \mathrm{UC}_{0.354}^{0.378} & \mathrm{UU}_{0.354}^{0.389} \\
& & \\
\mathrm{GC}_{0.193}^{0.197} & \mathrm{AC}_{0.175}^{0.118} & \mathrm{AU}_{0.175}^{0.112} \\
\mathrm{GG}_{0.060}^{0.065} & \mathrm{AG}_{0.028}^{0.048} & \mathrm{AA}_{-0.004}^{0.023}
\end{array}\right]
$$

\footnotetext{
${ }^{1)}$ В табл. 3 и далее в табл. 4,5 верхние и нижние значения относятся соответственно к экспериментальным и оценочным значениям.
} 
4.2. Гидрофобность. Подгоним теперь относительные гидрофобности $R_{x}$ шестнадшати динуклеозидных монофосфатов [6], используя четырехпараметрический оператор

$$
R_{x}=\alpha_{0}+\alpha_{1} J_{3 \mathrm{~V}}^{d}+\alpha_{2} J_{3 \mathrm{H}}^{d}+\alpha_{3}\left[\left(J_{3 \mathrm{H}}^{1}+J_{3 \mathrm{~V}}^{1}\right)^{2}+\left(J_{3 \mathrm{H}}^{2}+J_{3 \mathrm{~V}}^{2}\right)^{2}\right]
$$

(множитель при $\alpha_{3}$ равен двум для АA, AC, СА и СC, единице для $\mathrm{AU}, \mathrm{AG}, \mathrm{UA}, \mathrm{UC}, \mathrm{GC}$, $\mathrm{GA}, \mathrm{CU}$ и $\mathrm{CG}$ и нулю для UU, UG, GU и GG). C помощью метода наименьших квадратов получим наилучшее приближение для коэффициентов:

$$
\alpha_{0}=0.294, \quad \alpha_{1}=-0.240, \quad \alpha_{2}=-0.105, \quad \alpha_{3}=0.136 .
$$

Если же использовать подгонку по методу наименьших кваадратов, исключив из рассмотрения динуклеозид АА, то новые коэффициенты $\alpha_{i}$ будут давать лучшую оценку для величин $R_{x}$ оставшихся динуклеозидов:

$$
\alpha_{0}=0.309, \quad \alpha_{1}=-0.203, \quad \alpha_{2}=-0.068, \quad \alpha_{3}=0.099 .
$$

\section{ТАБЛИцА 4}

\section{Относительные гидрофобности $R_{x}$ динуклеозидов}

$$
\begin{gathered}
{\left[\begin{array}{r}
\mathrm{CA}_{0.507}^{0.494}
\end{array}\right] \quad\left[\begin{array}{rrr}
\mathrm{CG}_{0.340}^{0.326} & \mathrm{UG}_{0.309}^{0.291} & \mathrm{UA}_{0.476}^{0.441}
\end{array}\right]} \\
{\left[\begin{array}{r}
\mathrm{CU}_{0.205}^{0.218} \\
\mathrm{GU}_{0.309}^{0.291} \\
\mathrm{GA}_{0.611}^{0.660}
\end{array}\right] \quad\left[\begin{array}{rrr}
\mathrm{CC}_{0.236}^{0.244} & \mathrm{UC}_{0.205}^{0.218} & \mathrm{UU}_{0.174}^{0.194} \\
\mathrm{GC}_{0.340}^{0.326} & \mathrm{AC}_{0.507}^{0.494} & \mathrm{AU}_{0.476}^{0.441} \\
\mathrm{GG}_{0.444}^{0.436} & \mathrm{AG}_{0.611}^{0.660} & \mathrm{AA}_{0.778}^{1}
\end{array}\right]}
\end{gathered}
$$

Среднеквадратичное отклонение четырехпараметрической оценки (3) равно 0.049 . Среднеквадратичное отклонение оценки (4) равно 0.074 (если включать значение для AА) или 0.024 (если исключить коэффициент для АА). Экспериментальные и оценочные значения (оценка (4)) относительных гидрофобностей $R_{x}$ динуклеозидов собраны в табл. 4.

4.3. Свободная энергия. Особенно интересным представляется оценить значение свободной энергии, выделяемой при спаривании оснований при образовании двойной спирали РНК. Здесь данные получаются не для дублета нуклеотидов, каждый из которых лежит на своей нити спирали, а для пары соседних нуклеотидов (например, CG), лежащих на одной нити спирали и спаренных с дополнительной парой (т.е. GC) на противоположной нити. Отметим также, что направление спирали очень сушественно: выделение энергии для дублетной последовательности CG на первой нити, бегущей от 
$5^{\prime}$ до $3^{\prime}$ и спаренной с дублетом $\mathrm{GC}$ на противоположной нити, бегущей от $3^{\prime}$ до $5^{\prime}$, отличается от энергии, получаемой при спаривании дублета GC с дублетом CG. Кажется ясно, что подобные явления, связанные с упорядочением нуклеотидов в паре, должны относительно хорошо описываться естественным образом упорядоченным кристальным базисом, полученным из тензорного произведения двух представлений.

В работе [2] авторы использовали четырехпараметрический оператор для подгонки к экспериментальным данным. В данной статье более свежие экспериментальные данные [7] подгоняются с помошью двухпараметрического оператора, полученного из оператора работы [2] обрашением в нуль двух параметров:

$$
\Delta G_{37}^{0}=\alpha_{0}+\alpha_{1}\left(C_{\mathrm{H}}+C_{\mathrm{V}}\right) J_{3 \mathrm{H}}^{d} .
$$

Методом наименьших квадратов можно получить коэффициенты:

$$
\alpha_{0}=-2.14, \quad \alpha_{1}=-0.295 .
$$

Среднеквадратичное отклонение двухпараметрической оценки (5) равно 0.149. Экспериментальные и оценочные значения свободных энергий $\Delta G_{37}^{0}$ динуклеотидов представлены в табл 5 .

\section{ТАБЛИЦА 5}

Свободные энергии $\Delta G_{37}^{0}$ динуклеотидов

$$
\begin{aligned}
& {\left[\mathrm{CA}_{-2.14}^{-2.1}\right] \quad\left[\mathrm{CG}_{-2.73}^{-2.4} \quad \mathrm{UG}_{-2.14}^{-2.1} \quad \mathrm{UA}_{-1.55}^{-1.3}\right]} \\
& {\left[\begin{array}{c}
\mathrm{CU}_{-2.14}^{-2.1} \\
\mathrm{GU}_{-2.14}^{-2.2} \\
\mathrm{GA}_{-2.14}^{-2.4}
\end{array}\right]\left[\begin{array}{ccc}
\mathrm{CC}_{-3.32}^{-3.3} & \mathrm{UC}_{-2.14}^{-2.4} & \mathrm{UU}_{-0.96}^{-0.9} \\
\mathrm{GC}_{-3.32}^{-3.4} & \mathrm{AC}_{-2.14}^{-2.2} & \mathrm{AU}_{-0.96}^{-1.1} \\
\mathrm{GG}_{-3.32}^{-3.3} & \mathrm{AG}_{-2.14}^{-2.1} & \mathrm{AA}_{-0.96}^{-0.9}
\end{array}\right]}
\end{aligned}
$$

В более общем контексте, начиная с наблюдения о существовании взаимосвязи между кодонами и физико-химическими свойствами $(\Phi \mathrm{XC})$ соответствующих аминокислот [8], можно перейти к исследованию $\Phi Х С$ аминокислот в подходе модели кристального базиса; ФХС аминокислоты при этом зависят от следующих математических структур, представленных в порядке важности:

1) неприводимое представление (НП) динуклеотида, образованного первыми двумя нуклеотидами;

2) знак заряда $Q$ динуклеотидного состояния;

3) значение третьей компоненты $J_{3, \mathrm{~V}}$, если НП для динуклеотида уже фиксировано; 
4) верхние индексы НП кодона.

После этого можно получить набор корреляций и сравнить его с эспериментальными данными. Кроме того, можно предсказать значения термодинамических параметров трех аминокислот, которые еше не измерены экспериментально [9].

\section{5. КОРРЕЛЯЦИИ ПРИ ИСПОЛЬЗОВАНИИ КОДОНОВ [10], [11]}

Термин использование кодонов означает частотность использования данного кодона в биосинтезе всех белков. Определим вероятность использования кодона XYZ для данной аминокислоты как отношение между частотами появления кодона XYZ и появления $N$ копий соответствуюшей аминокислоты (т.е. как относительную вероятность данного кодона) в пределе больщих $N$. (Здесь и ниже буквы $\mathrm{X}, \mathrm{Y}, \mathrm{Z}, \mathrm{V}$ обозначают основания $\mathrm{C}, \mathrm{U}, \mathrm{G}, \mathrm{A}$.) Частотность использования кодона из мультиплета связана с условной вероятностью его использования $P(\mathrm{XYZ} \rightarrow$ А.к. $)$. Естественно предположить, что вероятность $P(\mathrm{XYZ} \rightarrow$ А.к. $)$ зависит от следующих факторов:

- биологический организм (б.о.), из которого извлекается указанная последовательность кодонов;

- анализируемая последовательность;

- природа соседних кодонов в цепочке;

- аминокислота;

- природа и структура мультиплета, ассоциированного с данной аминокислотой;

- биологическое окружение;

- свойства самого кодона (XYZ).

В дальнейшем пренебрежем временем протекания биосинтеза, т.е. будем предполагать, что весь биосинтез происходит одновременно, по крайней мере, по сравнению с временни́м масштабом эволюции генетического кода. Определим отношение расщеnления $B_{\mathrm{ZV}}$ как

$$
B_{\mathrm{ZV}}=\frac{P(\mathrm{XYZ} \rightarrow \text { А.к. })}{P(\mathrm{XYV} \rightarrow \text { А.к. })}
$$

Утверждение состоит в том, что для очень большого числа кодонов отношение расшепления в данном биологическом организме и для данной аминокислоты сушественно зависит от свойств кодонов. В указанной модели это означает, что в таком пределе $B_{\mathrm{ZV}}$ это функция, зависящая от типа мультиплета, от квантовых чиселкодонов XYZ и XYV (т.е. от значений $J_{\alpha}$ и $J_{\alpha}^{3}$ ) и, наконец, от другого набора квантовых чисел, снимающего вырождение по $J_{\alpha}$. В табл. 1 различные НП, отвечающие одному и тому же значению $J_{\alpha}$, различаются верхним индексом. Более того, будет предполагаться, что в указанном пределе $B_{\mathrm{ZV}}$ будет зависеть только от НП кодона, т.е.

$$
B_{\mathrm{ZV}}=F_{\mathrm{ZV}}(\text { б.о.; } \mathrm{H \Pi}(\mathrm{XYZ}) ; \mathrm{H \Pi}(\mathrm{XYV}))
$$


Отношение расщепления имеет смысл только для кодонов XYZ и XYV из одного и того же мультиплета, т.е. если они кодируют одну и ту же аминокислоту.

Рассмотрим квартеты и секстеты. В евкариотическом коде имеются пять квартетов и три секстета, что позволяет провести достаточно детальный анализ. Кроме того, каждый из этих трех секстетов представляется суммой квартета и дублета (см. табл. 1). В дальнейшем рассматриваются только квартетные составляющие секстетов. Пять аминокислот, закодированных квартетами, - это Pro, Ala, Thr, Gly и Val, а три аминокислоты, закодированные секстетами, - это Leu, Arg и Ser. B квартетах сушествуют шесть отношений расшеплений, из которых лиш три являются независимыми. Выберем отношения $B_{\mathrm{AG}}, B_{\mathrm{CG}}$ и $B_{\mathrm{UG}}$ как фундаментальные. Можно задать несколько функций $B_{\mathrm{ZV}}$ с отношениями вероятности для кодонов, отличающихся первыми двумя нуклеотидами $\mathrm{XY}$, т.е.

$$
\begin{aligned}
& B_{\mathrm{ZV}}=F_{\mathrm{ZV}}(\text { б.о.; } \mathrm{H \Pi}(\mathrm{XYZ}) ; \mathrm{H \Pi}(\mathrm{XYV})), \\
& B_{\mathrm{ZV}}^{\prime}=F_{\mathrm{ZV}}\left(\text { б.о.; } \mathrm{H \Pi}\left(\mathrm{X}^{\prime} \mathrm{Y}^{\prime} \mathrm{Z}\right) ; \mathrm{H} \Pi\left(\mathrm{X}^{\prime} \mathrm{Y}^{\prime} \mathrm{V}\right)\right) .
\end{aligned}
$$

При этом если обе пары кодонов XYZ, $\mathrm{X}^{\prime} \mathrm{Y}^{\prime} \mathrm{Z}$ и $\mathrm{XYV}, \mathrm{X}^{\prime} \mathrm{Y}^{\prime} \mathrm{V}$ принадлежат тому же самому НП, то

$$
B_{\mathrm{ZV}}=B_{\mathrm{ZV}}^{\prime}
$$

Был проведен анализ [12] набора экспериментальных данных, почерпнутых из банка информации “Таблицы использования кодонов по данным Генетического Банка" (Выпуск GenBank 119.0 от 15 августа 2000 г.). Были рассмотрены представители позвоночных, беспозвоночных и растений. Анализ в данной статье включает представителей 29 биологических организмов, пронумерованных от 1 (Homo sapiens) до 29 в табл. 6 ; число исследованных последовательностей и кодонов превосходит или примерно равно 200 и 100000 , соответственно [12].

Три квартета, кодируюшие аминокислоты Pro, Ala и Thr, имеют совершенно одинаковый состав НП (см. табл. 1). В табл. 6 собраны отношения расшепления $B_{\mathrm{AG}}$ для восьми аминокислот, закодированных квартетами и секстетами. Анализ этих данных показывает следующее:

- Наблюдается четкое соответствие между четырьмя аминокислотами Pro, Ala, Thr и Ser. Из табл. 1 следует, что НП в числителе отношений расшепления (см. (6)) для этих аминокислот всегда одно и то же: $(1 / 2,1 / 2)^{1}$ для $B_{\mathrm{AG}},(1 / 2,3 / 2)^{1}$ для $B_{\mathrm{UG}}$ и $(3 / 2,3 / 2)$ для $B_{\mathrm{CG}}$, в то время как НП в знаменателе - это $(1 / 2,1 / 2)^{1}$ для всех этих динуклеотидов. Относительные положения этих квартетов кодонов показаны на рисунке, где Pro, Ala, Thr и Ser (квартетная часть) образуют четыре ребра вертикальной колонны, связывающей представление $(1 / 2,1 / 2)^{1}$ на первом уровне сначала с представлением $(3 / 2,1 / 2)^{1}$, потом с представлением $(1 / 2,3 / 2)^{1}$ и, наконец, с представлением $(3 / 2,3 / 2)$ на верхнем уровне.

- Наблюдается явная коррелящия между двумя аминокислотами Val и Leu. Из табл. 1 видно, что НП в числителе формулы (6) для этих аминокислот также одно и то же: $(1 / 2,1 / 2)^{3}$ для $B_{\mathrm{AG}},(1 / 2,3 / 2)^{2}$ для $B_{\mathrm{UG}}$ и $(1 / 2,3 / 2)^{2}$ для $B_{\mathrm{CG}}$, в то 


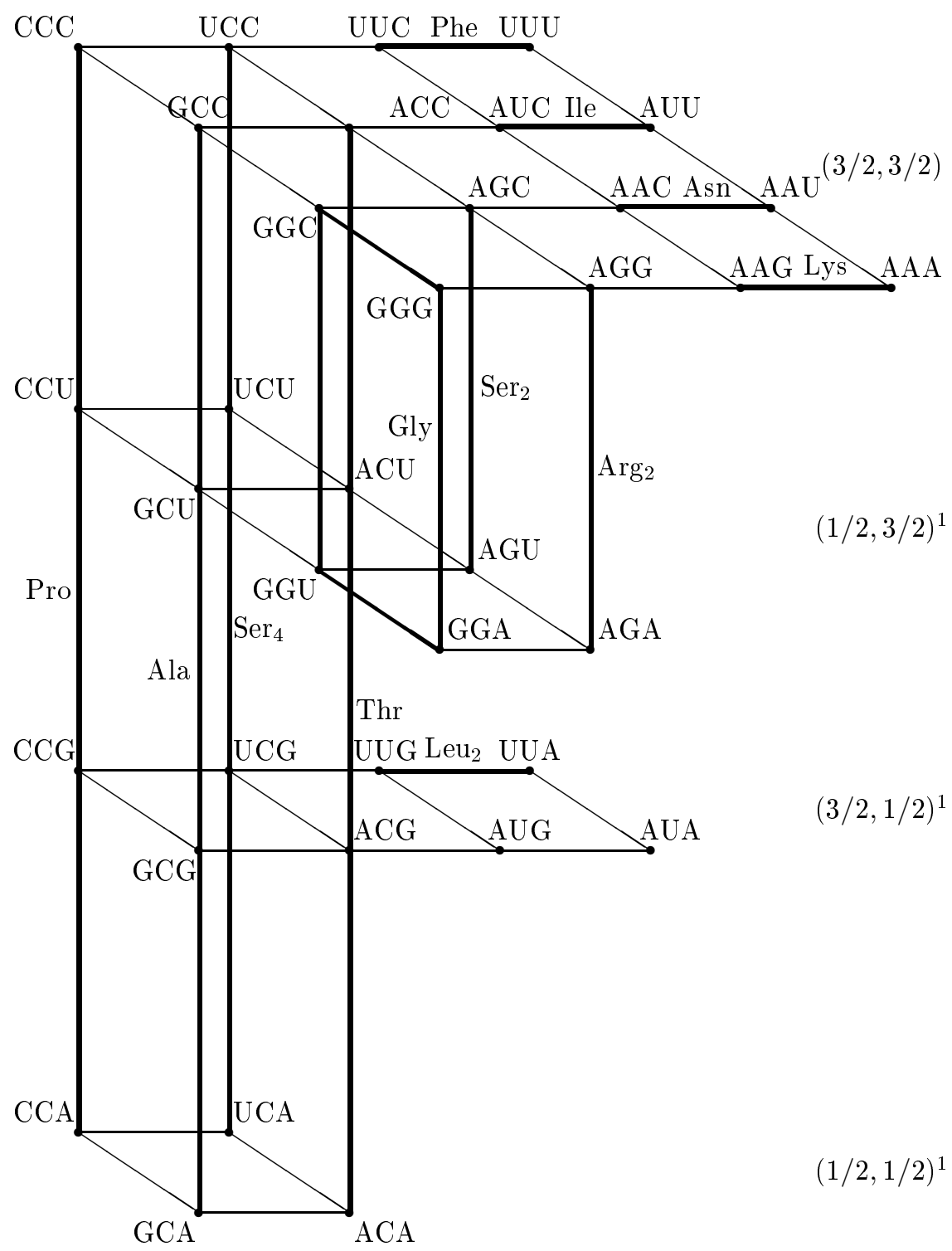

Классификация кодонов в различных кристальных базисах. 
Рисунок (продолжение)

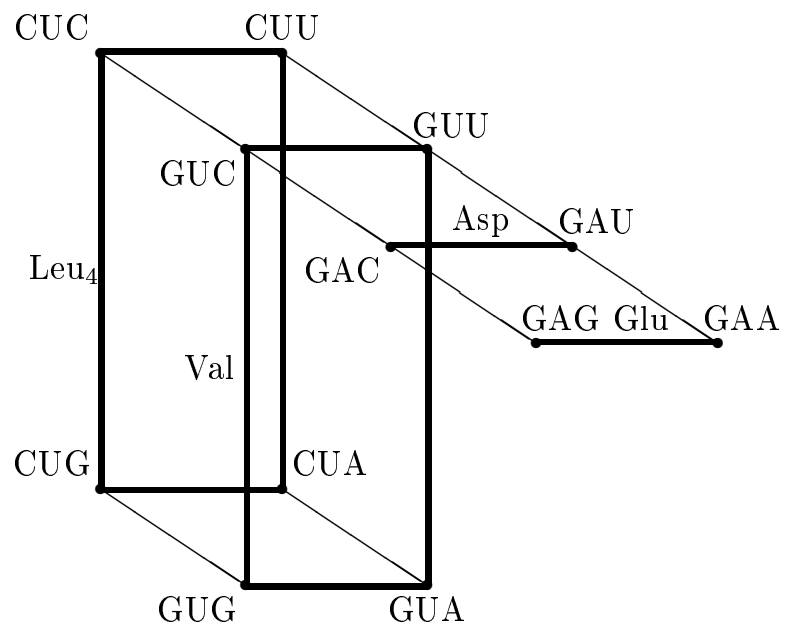

$(1 / 2,3 / 2)^{2}$

$(1 / 2,1 / 2)^{3}$

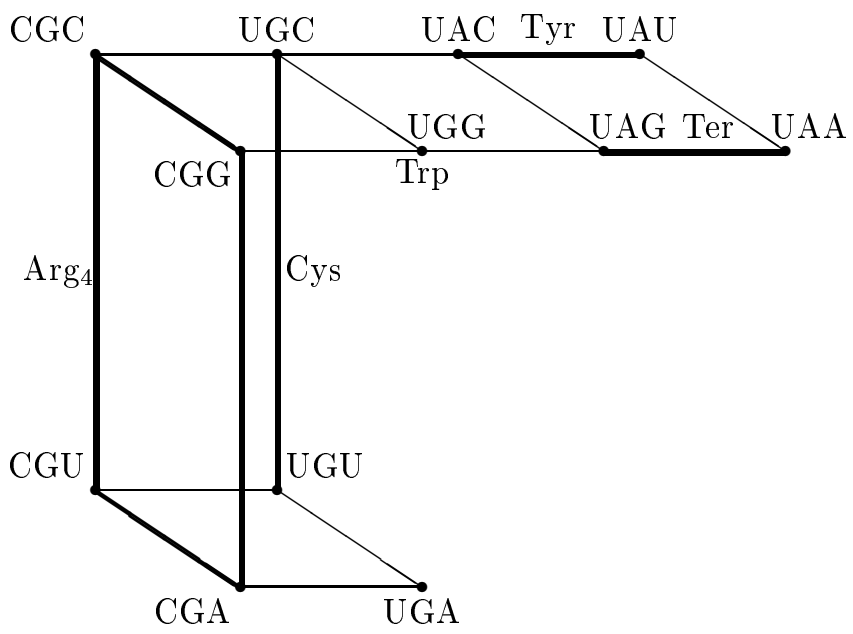

$(3 / 2,1 / 2)^{2}$

$(1 / 2,1 / 2)^{2}$

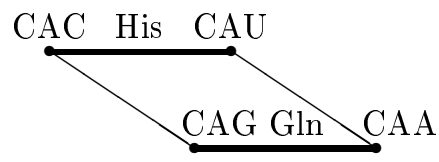

$(1 / 2,1 / 2)^{4}$ 
Отношения расщепления $B_{\mathrm{AG}}$

\begin{tabular}{|c|c|c|c|c|c|c|c|c|c|}
\hline & Биологический организм & Pro & Ala & Thr & Ser & Val & Leu & Arg & Gly \\
\hline 1 & Homo sapiens & 2.383 & 2.094 & 2.349 & 2.601 & 0.241 & 0.174 & 0.541 & 1.006 \\
\hline 2 & Bos aurus & 1.990 & 1.785 & 1.926 & 2.267 & 0.204 & 0.142 & 0.558 & 1.012 \\
\hline 3 & Sus scrofa & 1.772 & 1.685 & 1.673 & 1.913 & 0.183 & 0.166 & 0.500 & 0.955 \\
\hline 4 & Ovis aries & 2.027 & 1.622 & 1.841 & 1.982 & 0.166 & 0.143 & 0.627 & 1.108 \\
\hline 5 & Canis familiaris & 2.150 & 1.710 & 1.945 & 2.036 & 0.221 & 0.154 & 0.493 & 1.046 \\
\hline 6 & Oryctolagus cuniculus & 1.409 & 1.421 & 1.298 & 1.421 & 0.148 & 0.102 & 0.438 & 0.879 \\
\hline 7 & Rattus norvegicus & 2.433 & 2.195 & 2.363 & 2.383 & 0.227 & 0.174 & 0.622 & 1.043 \\
\hline 8 & Rattus sp. & 2.229 & 2.119 & 2.271 & 2.182 & 0.207 & 0.165 & 0.630 & 1.058 \\
\hline 9 & Gallus gallus & 1.763 & 1.900 & 1.937 & 1.907 & 0.250 & 0.140 & 0.506 & 1.015 \\
\hline 10 & Xenopus laevis & 4.119 & 4.183 & 4.100 & 3.412 & 0.479 & 0.315 & 0.991 & 1.672 \\
\hline 11 & Danio rerio & 1.507 & 1.578 & 1.820 & 1.826 & 0.227 & 0.164 & 0.910 & 2.062 \\
\hline 12 & Drosophila melanogaster & 0.836 & 0.914 & 0.747 & 0.459 & 0.223 & 0.212 & 1.014 & 3.729 \\
\hline 13 & Bombyx mori & 0.862 & 0.998 & 1.239 & 1.318 & 0.612 & 0.455 & 1.214 & 3.743 \\
\hline 14 & Caenorhabditis elegans & 2.719 & 2.435 & 2.262 & 1.722 & 0.697 & 0.666 & 2.525 & 6.937 \\
\hline 15 & Leishmania major & 0.392 & 0.462 & 0.396 & 0.337 & 0.136 & 0.118 & 0.496 & 0.541 \\
\hline 16 & Trypanosoma cruzi & 0.760 & 0.611 & 0.561 & 0.693 & 0.153 & 0.119 & 0.665 & 0.826 \\
\hline 17 & Trypanosoma brucei & 1.249 & 1.315 & 1.224 & 1.253 & 0.467 & 0.478 & 0.733 & 1.374 \\
\hline 18 & Arabidopsis thaliana & 1.949 & 2.023 & 2.081 & 2.019 & 0.591 & 1.006 & 1.305 & 2.322 \\
\hline 19 & Zea mays & 0.958 & 0.731 & 0.925 & 1.035 & 0.214 & 0.252 & 0.406 & 0.898 \\
\hline 20 & Oryza sativa & 0.736 & 0.608 & 0.897 & 0.925 & 0.268 & 0.328 & 0.504 & 0.904 \\
\hline 21 & Hordeum vulgare & 0.709 & 0.552 & 0.568 & 0.689 & 0.148 & 0.196 & 0.262 & 0.697 \\
\hline 22 & Triticum aestivum & 1.530 & 0.698 & 0.949 & 1.135 & 0.233 & 0.307 & 0.426 & 0.905 \\
\hline 23 & Glycine $\max$ & 5.063 & 3.811 & 3.655 & 3.189 & 0.330 & 0.658 & 1.359 & 1.747 \\
\hline 24 & Pisum sativum & 3.762 & 3.961 & 4.441 & 3.785 & 0.556 & 1.090 & 1.638 & 2.889 \\
\hline 25 & Nicotiana tabacum & 4.408 & 3.924 & 4.026 & 3.337 & 0.656 & 0.845 & 1.522 & 2.327 \\
\hline 26 & Solanum tuberosum & 5.232 & 5.156 & 5.029 & 4.137 & 0.654 & 1.024 & 1.984 & 2.707 \\
\hline 27 & Lycopersicon esculentum & 4.654 & 4.484 & 3.805 & 3.843 & 0.659 & 1.036 & 1.790 & 2.538 \\
\hline 28 & Brassica napus & 1.900 & 1.807 & 1.746 & 1.828 & 0.405 & 0.927 & 1.242 & 2.594 \\
\hline 29 & Chlamydomonas reinhardtii & 0.239 & 0.235 & 0.226 & 0.141 & 0.041 & 0.036 & 0.190 & 0.472 \\
\hline
\end{tabular}

время как НП в знаменателе равно $(1 / 2,1 / 2)^{3}$. Два представления $(1 / 2,1 / 2)^{3}$ и $(1 / 2,3 / 2)^{2}$ приведены на рисунке, при этом кодоны, связанные с аминокислотами Val и Leu (с их квартетными составляюшими), образуют вершины двух параллельных параллелограммов.

- Не наблюдается корреляции Arg и Gly с другими аминокислотами, что находится в соответствии с присваиванием НП в табл. 1. В самом деле, заметим, что представления $(1 / 2,1 / 2)^{2}$ и $(3 / 2,1 / 2)^{2}$ на рисунке связаны только лишь через кодонный квартет, ассоциированный с Arg. Отметим также, что квартет Gly участвует в представлениях $(1 / 2,3 / 2)^{1}$ и $(3 / 2,3 / 2)$; его положение тем самым совершенно отлично от всех вышеперечисленных квартетов.

Мы не обсуждали здесь отношения расщепления $B_{\mathrm{UG}}$ и $B_{\mathrm{CG}}$ (см. [11]). Другие интересные результаты, получаемые из модели кристального базиса, можно найти в работе [4]. 


\section{6. ОБСУЖДЕНИЕ}

Математическая составляюшая исследуемой модели, описывающей генетический код, - это квантовая алгебра в пределе $q \rightarrow 0$. Этот подход обосновывается с помощью некоторых приложений. Первое исследование мутаций содержится в работе [4]. Отметим, что интерпретация мРНK в Physarum polycephalum в терминах принципа минимальности была предложена в работе [13].

Среди многих важных вопросов, остающихся открытыми, отметим проблему эволюции, которая может быть математически исследована с помошью оператора считьвания (см. раздел 3). Кроме того, исследуемая модель нуждается в улучшении, в частности надо уметь учитывать взаимодействия меж ду нуклеотидами на одной и той же нити спирали ДНК. Интересно интерпретировать двойную спираль как двойную спиновую цепочку.

Благодарности. Один из авторов (П. С.) искренне признателен организаторам конференции NEEDS 2000 в Гокове.

\section{Список литературы}

[1] M. Singer, P. Berg. Genes and Genomes. Paris: Editions Vigot, 1992.

[2] L. Frappat, A. Sciarrino, P. Sorba. Phys. Lett. A. 1998. V. 250. P. 214; physics/9801027.

[3] M. Kashiwara. Commun. Math. Phys. 1990. V. 133. P. 249.

[4] L. Frappat, A. Sciarrino, P. Sorba. Crystalizing the genetic code. To appear in J. Biological Phys.; physics/0003037.

[5] A. L. Weber, J. C. Lacey. J. Mol. Evol. 1978. V. 11. P. 199.

[6] J. R. Jemcyck. J. Mol. Evol. 1978. V. 11. P. 211.

[7] D. H. Mathews, J. Sabina, M. Zucker, D. H. Turner. J. Mol. Biol. 1999. V. 288. P. 911.

[8] M. Sjostrom and S. Wold. J. Mol. Evol. 1985. V. 22. P. 272.

[9] L. Frappat, A. Sciarrino, P. Sorba. Theoretical predictions of physico-chemical properties of amino acids from genetic code. Preprint LAPTH-805/00, 2000; physics/0007034.

[10] L. Frappat, A. Sciarrino, P. Sorba. Phys. Lett. A. 1999. V. 259. P. 339; physics/9812041.

[11] M. L. Chiusano, L. Frappat, A. Sciarrino, P. Sorba. Codon usage correlations and crystal basis model of the genetic code. To appear in Europhys. Lett.

[12] Y. Nakamura, T. Gojobori, T. Ikemura. Nucleic Acids Research. 1998. V. 26. P. 334.

[13] L. Frappat, A. Sciarrino, P. Sorba. A minimum principle in mRNA editing of Physarum. Preprint LAPTH-812/00, 2000; physics/0009063. 